\title{
Confining and repulsive potentials from effective non-Abelian gauge fields in graphene bilayers
}

\author{
J. González \\ Instituto de Estructura de la Materia, Consejo Superior de Investigaciones Científicas, Serrano 123, 28006 Madrid, Spain
}

(Received 29 July 2016; published 3 October 2016)

\begin{abstract}
We investigate the effect of shear and strain in graphene bilayers, under conditions where the distortion of the lattice gives rise to a smooth one-dimensional modulation in the stacking sequence of the bilayer. We show that strain and shear produce characteristic Moiré patterns which can have the same visual appearance on a large scale, but representing graphene bilayers with quite different electronic properties. The different features in the low-energy electronic bands can be ascribed to the effect of a fictitious non-Abelian gauge field mimicking the smooth modulation of the stacking order. Strained and sheared bilayers show a complementary behavior, which can be understood from the fact that the non-Abelian gauge field acts as a repulsive interaction in the former, expelling the electron density away from the stacking domain walls, while behaving as a confining interaction leading to localization of the electronic states in the sheared bilayers. In this latter case, the presence of the effective gauge field explains the development of almost flat low-energy bands, resembling the form of the zeroth Landau level characteristic of a Dirac fermion field. The estimate of the gauge field strength in those systems gives a magnitude of the order of several tens of tesla, implying a robust phenomenology that should be susceptible of being observed in suitably distorted bilayer samples.
\end{abstract}

DOI: 10.1103/PhysRevB.94.165401

\section{INTRODUCTION}

During more than a decade, graphene has been attracting much attention due to its vast potential for technological application. The one-atom-thick carbon layer displays many unconventional electronic properties, that derive to a great extent from the peculiar conical dispersion of its valence and conduction bands [1-3]. The behavior of the electron quasiparticles in graphene is similar to that of relativistic massless fermions, which explains the appearance of phenomena like the Klein paradox [4] and the limited backscattering experienced by electrons in the material.

A remarkable feature of graphene is also that the interplay between the electronic degrees of freedom and the geometry of the lattice makes it possible to mimic the effect of gauge fields acting on the electron quasiparticles. This connection goes back to the description of the fullerene lattices, where it has been shown that the degeneracies in the low-energy electronic spectrum may be understood from the action of a fictitious non-Abelian gauge field, induced by the pentagonal rings in the lattice [5]. The consistency of the gauge field construction is certified in that case by the correspondence between the degeneracy of the low-lying electronic levels and the total flux of the effective magnetic field traversing the surface of the molecule.

More recently, the study of the effects of strain on the electron quasiparticles has unveiled the possibility of engineering a fictitious gauge field in the graphene lattice [6]. The distortions of the honeycomb lattice can play a role similar to that of a real gauge field, shifting locally the Dirac cones in momentum space $[7,8]$. It has been shown that some configurations may actually give rise to an effective magnetic field, with a sequence of Landau levels resembling that from a real transverse magnetic field [6]. This has been experimentally confirmed when looking at the nanobubbles which form in some graphene samples on a substrate, finding signatures of effective magnetic fields with magnitudes of the order of $\sim 300$ T [9].
Yet a number of different effects have been related to the appearance of fictitious gauge fields in graphene bilayers [10-13]. In these systems, a small amount of strain or shear may give rise to a deviation with respect to the perfect registry corresponding to Bernal stacking (so-called $A B$ stacking) of conventional bilayer graphene. When the lattice distortion takes the form of a smooth one-dimensional modulation of the stacking sequence, the changes induced in the low-energy electronic spectrum can be understood as arising from an effective non-Abelian gauge field acting on the internal space of two Dirac cones (one for each carbon layer) [12,14]. It has been proposed indeed that some configurations with alternating $A B-B A$ stacking sequence may lead to largely degenerate electronic levels, extending into linear branches of edge states and thus providing a clear analogy with the physics of the quantum Hall effect [12].

The present paper is devoted to discriminate the effect of the fictitious non-Abelian gauge fields in different graphene bilayers with smooth domain walls between $A B$ and $B A$ stacking. In this respect, we note that a sequence of such stacking regions can be formed by applying either shear or strain, or in general a combination of both. This leads to stacking sequences with Moiré patterns which may have the same appearance from a wide perspective, but representing graphene bilayers with quite different electronic properties. Thus, when the stacking sequence is created by shear, we will see that the non-Abelian gauge field manifests as a confining interaction, leading to localization of electronic states and the development of almost flat low-energy bands. When the stacking sequence is created instead by applying strain (pulling in a direction perpendicular to the hexagon rows in the honeycomb lattice), we will find that the low-energy electronic states feel a repulsive potential arising from the non-Abelian gauge field, showing no sign of localization in the band structure.

Our analysis becomes relevant as several experimental observations have already shown domain walls between regions of different stacking order in graphene bilayers [15-19]. 
There have been theoretical studies showing that such domain walls may support one-dimensional electronic states, once the bilayer electron system is gapped in the bulk by applying a transverse electric field [20,21]. It has been found that such states behave much in the same way as those arising at domain walls induced by a change in the interlayer electric field [22,23]. Regarding our investigation, the difference with respect to these studies is that here we address the genuine effects of the non-Abelian gauge field characterizing smooth domain walls between $A B$ and $B A$ stacking, when no transverse electric field is applied to the bilayer. This description may become particularly suitable in those cases where the transition between different domains is not abrupt, which seems to be common as typical atomic-scale images of the stacking domain walls show widths of the order of $\sim 10$ $\mathrm{nm}$.

One of the main findings of our work is that an effective non-Abelian gauge field may lead to the development of a zeroth Landau level which is quite similar to that arising from the effect of a real transverse magnetic field on Dirac quasiparticles. The counterpart to this remark is that not all the configurations of the non-Abelian gauge field share such a flat band characteristic of Landau quantization. This establishes a clear difference with respect to the action of an Abelian gauge field, leading to electronic features which are nicely illustrated in graphene bilayers with different stacking domain walls.

\section{ONE-DIMENSIONAL MOIRÉ PATTERNS IN STRAINED AND SHEARED GRAPHENE BILAYERS}

When shear or strain is applied to the sheets of a graphene bilayer, a Moiré pattern in general appears, which is the reflection of a sequence of regions alternating between $A B$ and $B A$ stacking (the conventional stacking in bilayer graphene) and $A A$ stacking (where homologous points in the two layers fall one on top of each other). We are going to concentrate here on patterns where the alternation takes place along two different orthogonal directions, which are perpendicular in one case and aligned in the other with respect to the hexagon rows in the original layers.

An alternating sequence of $A B, B A$, and $A A$ stacking can be obtained by applying shear, by means for instance of a lateral shift at the border of one of the layers, producing the pattern shown in Fig. 1(a). But there is another possibility to produce a sequence of stacking regions by applying tensile strain in one of the sheets, in a direction perpendicular to the hexagon rows of the honeycomb lattice, leading to the pattern shown in Fig. 1(b). We observe that the Moiré patterns shown in Figs. 1(a) and 1(b) have the same appearance on a large scale, although the distortions producing them are quite different at the atomic scale. In general, we may think of other Moiré patterns with alternating stacking as the result of combining strain and shear [24], leading to one-dimensional periodic sequences along directions which do not coincide with the principal axes of the original bilayer.

The bilayers shown in Figs. 1(a) and 1(b) can be then characterized by the different strain supported by the respective lattices. This can be quantified in terms of the strain tensors of

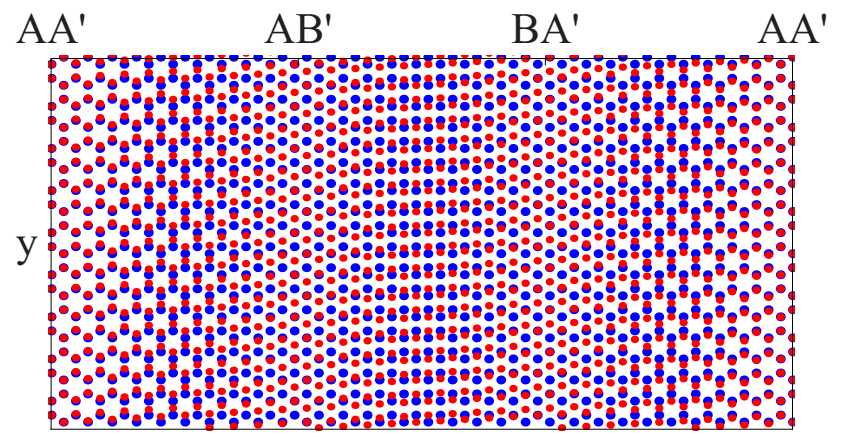

$\mathrm{X}$

(a)

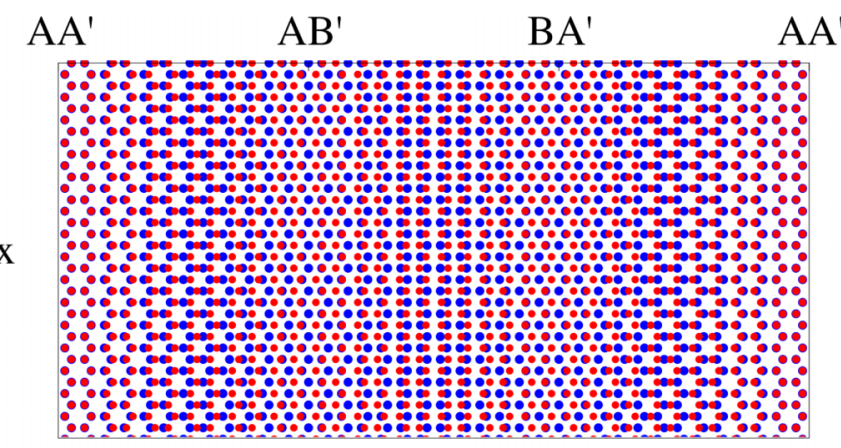

$\mathrm{y}$

(b)

FIG. 1. Different types of Moiré patterns in graphene bilayers, which can be obtained (a) by means of shear, pulling laterally the layers in opposite directions, and (b) by applying tensile strain, pulling the layers in the direction perpendicular to the hexagon rows in the honeycomb lattice.

the two layers (labeled by $a=1,2$ )

$$
u_{i j}^{(a)}=\frac{1}{2}\left(\partial_{i} u_{j}^{(a)}+\partial_{j} u_{i}^{(a)}+\partial_{i} h^{(a)} \partial_{j} h^{(a)}\right),
$$

where $\left(u_{x}^{(a)}, u_{y}^{(a)}, h^{(a)}\right)$ denotes the three-dimensional displacement field of the layer with respect to the equilibrium position. Thus the bilayer shown in Fig. 1(a) has a strain configuration with

$$
u_{x y}^{(a)} \neq 0
$$

On the other hand, the bilayer in Fig. 1(b) corresponds to the case with

$$
u_{y y}^{(a)} \neq 0
$$

Strain fields with constant strain tensors given by (2) or (3) can already induce slight modifications in the low-energy electronic bands of the individual layers. We recall that the effect of strain can be mimicked by the action of a gauge field on the electron quasiparticles of graphene. The correspondence is such that a strain field $u_{i}^{(a)}$ has the same effect as a vector potential given by [8]

$$
\tilde{\mathbf{A}}^{(a)}=\frac{\beta}{a}\left(\begin{array}{c}
u_{x x}^{(a)}-u_{y y}^{(a)} \\
2 u_{x y}^{(a)}
\end{array}\right),
$$


where $a$ stands for the $\mathrm{C}-\mathrm{C}$ distance and $\beta$ represents the variation of the tunneling amplitude with respect to the lattice spacing. Then, the bilayer in Fig. 1(a) is characterized by having an effective vector potential with $\tilde{A}_{y}^{(a)} \neq 0$, while the bilayer in Fig. 1(b) has instead $\tilde{A}_{x}^{(a)} \neq 0$. The patterns shown in Figs. 1(a) and 1(b) can be obtained with just a constant $u_{x y}^{(a)}$ or $u_{y y}^{(a)}$. In this case, the strain configurations correspond to vanishing pseudomagnetic field, and their effect can be seen as a shift of the Dirac cones representing the low-energy electronic states.

The important changes in the band structure of the bilayers come however from the modulation of the stacking order. In this regard, a remarkable observation is that the low-energy bands of the bilayers shown in Figs. 1(a) and 1(b) have completely different shapes. To illustrate this fact, we can rely on a tight-binding approximation, taking into account intralayer and interlayer tunneling amplitudes to express the Hamiltonian of a graphene bilayer in the form

$$
H_{\mathrm{tb}}=-\sum_{i, j} t_{i j} a_{i}^{\dagger} a_{j}-\sum_{i, j} t_{i j} b_{i}^{\dagger} b_{j}-\sum_{i, j} \tilde{t}_{i j} a_{i}^{\dagger} b_{j}
$$

in terms of electron creation (annihilation) operators $a_{i}^{\dagger}\left(a_{i}\right)$ for the different sites of the upper layer, and similar operators $b_{i}^{\dagger}, b_{i}$ for the lower layer. In (5), one can assume an exponential decay to represent the interlayer tunneling amplitudes between sites with variable separation [25]

$$
\tilde{t}_{i j}\left(\mathbf{r}_{i}-\mathbf{r}_{j}\right)=t_{0} e^{-\left|\mathbf{r}_{i}-\mathbf{r}_{j}\right| / a_{0}} .
$$

We have chosen in particular $t_{0}$ and $a_{0}$ so that the hopping parameter between nearest-neighbor sites in each carbon layer is set to $3.2 \mathrm{eV}$, while the hopping parameter between nearestneighbor sites in different layers (in the region of $A B$ stacking) is set to $0.3 \mathrm{eV}$. In practice, we have reduced the complexity of the model by restricting intralayer hopping to nearest-neighbor sites, and taking a finite range $r_{0}$ for the interlayer tunneling to allow hopping up to a distance equal to the next-to-nearestneighbor separation (in the region of $A B$ stacking) between different layers.

We can see for instance in Figs. 2(a) and 2(b) the low-energy bands obtained with the tight-binding approach for a bilayer of the type shown in Fig. 1(a), with infinite length in the $y$ direction and length $L=210 \sqrt{3} a$ ( $a$ being the C-C distance) in the $x$ direction. The two different plots correspond to taking periodic [Fig. 2(a)] and open [Fig. 2(b)] boundary conditions. In the case of a bilayer of the type shown in Fig. 1(b), results from the tight-binding calculation are represented in Figs. 2(c) and 2(d), for a geometry with infinite length in the $x$ direction and length $L=633 a$ in the $y$ direction. The plots show in this case the low-energy bands developing about one of the Dirac valleys, while a similar structure (related by mirror symmetry) is to be found at the opposite Dirac valley. The two representations differ in the choice of periodic [Fig. 2(c)] and open [Fig. 2(d)] boundary conditions.

We note that the details of the bands shown in Figs. 2(a)2(d) may slightly depend on the particular range taken for the interlayer tunneling. However, it can be checked that the most salient qualitative features, like the existence of almost flat bands close to zero energy for the sheared bilayer or the parabolic bands for the strained bilayer, persist

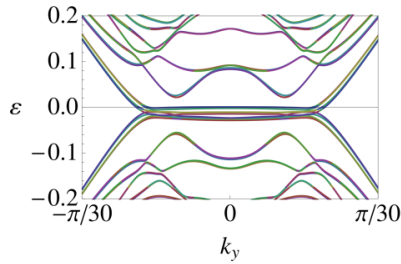

(a)

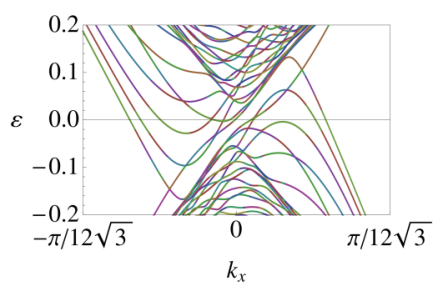

(c)

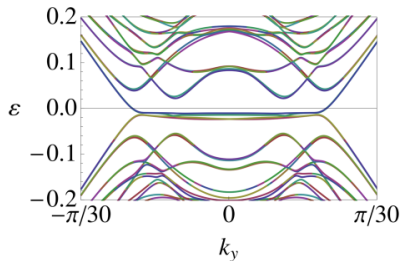

(b)

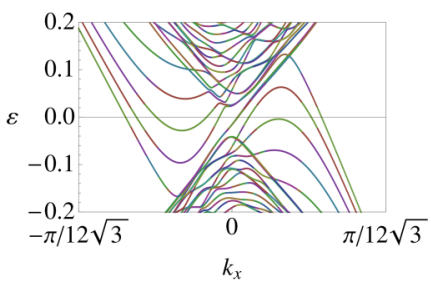

(d)
FIG. 2. (a), (b) Low-energy bands around the Fermi level of an undoped bilayer of the type shown in Fig. 1(a), obtained by means of a tight-binding approximation for a geometry of infinite length along the vertical direction and length $L=210 \sqrt{3} a$ ( $a$ being the C-C distance) along the horizontal direction, with periodic (a) and open (b) boundary conditions. (c), (d) Plots obtained in similar fashion as (a), (b), representing the low-energy bands (from a given Dirac valley) of a graphene bilayer of the type shown in Fig. 1(b), for a geometry of infinite length along the vertical direction and length $L=633 a$ along the horizontal direction with periodic (c) and open (d) boundary conditions. The energy $\varepsilon$ is given in $\mathrm{eV}$ and the momentum in units of the inverse of the $\mathrm{C}-\mathrm{C}$ distance.

under variations of the parameters used in the tight-binding resolution. In this respect, it becomes clear that the effects of strain or shear within each layer cannot have a significant impact on the electron system at large $L$, since the large period of the Moiré pattern implies that it must be produced by a small distortion with $u_{x y}^{(a)}$ or $u_{y y}^{(a)}$ of order $\sim 1 / L$. The great contrast between the bands in Figs. 2(a) and 2(b) and those in Figs. 2(c) and 2(d) must be therefore the consequence of some other effect different to that associated to the effective gauge field $\tilde{A}_{i}^{(a)}$.

As we are going to see, the generic features that appear in the low-energy bands of strained and sheared bilayers can be explained from the existence of a different type of effective gauge field, which can be recognized in the continuum limit approximation to the electron system. This large- $L$ approach will allow us to understand why the band structures of the strained and the sheared bilayers are so different from each other, despite the similar visual appearance for large period of the Moiré patterns.

\section{MODEL OF EFFECTIVE NON-ABELIAN GAUGE FIELDS}

We start by assuming that, in each individual layer (when the interlayer coupling is ideally switched off), the low-energy dynamics of electron quasiparticles is governed by a Dirac Hamiltonian (focusing on a given Dirac valley)

$$
H^{(a)}=v_{F} \boldsymbol{\sigma} \cdot\left(-i \boldsymbol{\partial}-\tilde{\mathbf{A}}^{(a)}\right)
$$


with the parameter $v_{F}$ standing for the Fermi velocity. Moreover, we focus on graphene bilayers having Moiré patterns with fairly large period, for which the coupling between carbon layers can be seen as a smooth spatial modulation of the interlayer tunneling. In general, we may discern between different interlayer amplitudes depending on the sublattices
$A$ and $B$ of each graphene lattice, and evolving from one stacking domain to the next. A simple model can be built by assembling the Dirac quasiparticles from the two layers into a four-component spinor $\Psi=\left(\psi_{A}^{(1)}, \psi_{B}^{(1)}, \psi_{A^{\prime}}^{(2)}, \psi_{B^{\prime}}^{(2)}\right)$. In this representation, the Hamiltonian accounting for the tunneling between layers can be written as

$$
H=v_{F}\left(\begin{array}{cccc}
0 & -i \nabla_{x}^{(1)}-\nabla_{y}^{(1)} & V_{A A^{\prime}}(\mathbf{r}) & V_{A B^{\prime}}(\mathbf{r}) \\
-i \nabla_{x}^{(1)}+\nabla_{y}^{(1)} & 0 & V_{B A^{\prime}}(\mathbf{r}) & V_{A A^{\prime}}(\mathbf{r}) \\
V_{A A^{\prime}}^{\star}(\mathbf{r}) & V_{B A^{\prime}}^{\star}(\mathbf{r}) & 0 & -i \nabla_{x}^{(2)}-\nabla_{y}^{(2)} \\
V_{A B^{\prime}}^{\star}(\mathbf{r}) & V_{A A^{\prime}}^{\star}(\mathbf{r}) & -i \nabla_{x}^{(2)}+\nabla_{y}^{(2)} & 0
\end{array}\right),
$$

where we have introduced the covariant derivatives $\nabla_{i}^{(a)} \equiv$ $\partial_{i}-i \tilde{A}_{i}^{(a)}$, and $V_{A A^{\prime}}, V_{A B^{\prime}}, V_{B A^{\prime}}$ stand for the interlayer tunneling amplitudes between different sublattices.

An important observation is that, assuming that the interlayer potentials are real functions, they can be written in terms of gauge fields with off-diagonal action on the Dirac quasiparticles of the bilayer [12]. We can introduce the fields $A_{x}$ and $A_{y}$ according to the decomposition

$$
\begin{aligned}
& V_{A B^{\prime}}(\mathbf{r})=-A_{x}(\mathbf{r})+A_{y}(\mathbf{r}), \\
& V_{B A^{\prime}}(\mathbf{r})=-A_{x}(\mathbf{r})-A_{y}(\mathbf{r}) .
\end{aligned}
$$

Then we can recast the Hamiltonian (8) by thinking of the field $A_{x}$ as an off-diagonal shift of the momentum operator $-i \nabla_{x}$, and assigning the same role for $A_{y}$ with respect to the operator $-i \nabla_{y}$. We can write

$$
H=v_{F} \boldsymbol{\sigma} \cdot(-i \nabla-\hat{\mathbf{A}})+v_{F} V_{A A^{\prime}} \tau_{1}
$$

introducing the vector potential

$$
\hat{\mathbf{A}}=\left(\begin{array}{l}
A_{x} \tau_{1} \\
A_{y} \tau_{2}
\end{array}\right)
$$

in terms of a new set of Pauli matrices $\left\{\tau_{i}\right\}$ acting on the internal space of the two layers.

The representation (11) highlights that $\hat{\mathbf{A}}$ is indeed a nonAbelian gauge field, as its associated gauge transformations are valued in the group $S U(2)$. This has also a reflection in the dynamics of the Dirac quasiparticles. Disregarding for simplicity the scalar potential $V_{A A^{\prime}}$ at this point, we can take the square of the Hamiltonian (11) to end up with the eigenvalue equation

$$
v_{F}^{2}\left((-i \nabla-\hat{\mathbf{A}})^{2}-\sigma_{z} \hat{F}_{x y}\right) \Psi=\varepsilon^{2} \Psi,
$$

where the field strength of the non-Abelian gauge potential is [26]

$$
\hat{F}_{i j}=\nabla_{i} \hat{A}_{j}-\nabla_{j} \hat{A}_{i}-i\left[\hat{A}_{i}, \hat{A}_{j}\right] .
$$

In Eq. (14), $\hat{A}_{i}$ stands for the matrix-valued vector potential. The last term of the field strength with the commutator provides actually the relevant contribution in the Moiré bilayers for large period $L$, since the derivatives of the gauge field become then of order $\sim 1 / L$ and are therefore subdominant in that limit.

\section{A. Confinement from non-Abelian gauge fields in sheared bilayers}

We pay attention first to the case in which shear with constant $u_{x y}^{(a)} \neq 0$ is responsible for the formation of a Moiré pattern like that in Fig. 1(a). In the limit of large $L$, we may consider the interlayer potentials $V_{A A^{\prime}}, V_{A B^{\prime}}$, and $V_{B A^{\prime}}$ as smooth functions varying only along the $x$ direction. Moreover, we can also neglect terms with derivatives of the gauge field $\hat{A}_{i}$, which give subdominant contributions of order $\sim 1 / L$. To carry out the analysis of the effects of the non-Abelian gauge field, we may concentrate on the eigenvalue problem (13). Introducing solutions of the form

$$
\Psi(\mathbf{r})=e^{i k_{y} y} \chi(x),
$$

we get at large $L$

$$
\begin{gathered}
v_{F}^{2}\left(\left(-i \partial_{x}-A_{x}(x) \tau_{1}\right)^{2}+\left(k_{y}-A_{y}(x) \tau_{2}\right)^{2}\right. \\
\left.-2 \sigma_{z} \tau_{3} A_{x}(x) A_{y}(x)\right) \chi(x)=\varepsilon^{2} \chi(x) .
\end{gathered}
$$

Given that the effective gauge field depends only on the $x$ variable, it is possible to partially integrate out $A_{x}$ from (16) by applying a gauge transformation $\chi=U \tilde{\chi}$ with

$$
\begin{gathered}
U=\exp \left(i \theta(x) \tau_{1}\right), \\
\theta(x)=\int^{x} d s A_{x}(s) .
\end{gathered}
$$

The eigenvalue equation becomes then

$$
\begin{aligned}
& v_{F}^{2}\left(-\partial_{x}^{2}+U^{\dagger}\left(k_{y}-A_{y}(x) \tau_{2}\right)^{2} U\right. \\
& \left.\quad-2 \sigma_{z} U^{\dagger} \tau_{3} A_{x}(x) A_{y}(x) U\right) \tilde{\chi}(x)=\varepsilon^{2} \tilde{\chi}(x) .
\end{aligned}
$$

Working out the algebra of Pauli matrices, we get from (19)

$$
\begin{aligned}
& v_{F}^{2}\left(-\partial_{x}^{2}+\left(k_{y}-A_{y}(x) \hat{n}\right)^{2}+\left(A_{y}(x)-\sigma_{z} A_{x}(x) \hat{m}\right)^{2}\right. \\
& \left.\quad-A_{x}^{2}(x)-A_{y}^{2}(x)\right) \tilde{\chi}(x)=\varepsilon^{2} \tilde{\chi}(x),
\end{aligned}
$$

where we have the matrices with unit square

$$
\begin{gathered}
\hat{m}=\cos (2 \theta(x)) \tau_{3}-\sin (2 \theta(x)) \tau_{2}, \\
\hat{n}=\sin (2 \theta(x)) \tau_{3}+\cos (2 \theta(x)) \tau_{2} .
\end{gathered}
$$

The advantage of the expression (20) is that it can be interpreted as a Schrödinger equation, from which an effective 
potential $V_{\text {eff }}(x)$ can be read in terms of the components of the gauge field

$$
\begin{aligned}
V_{\mathrm{eff}}(x)= & \left(k_{y}-A_{y}(x) \hat{n}\right)^{2}+\left(A_{y}(x)-\sigma_{z} A_{x}(x) \hat{m}\right)^{2} \\
& -A_{x}^{2}(x)-A_{y}^{2}(x) .
\end{aligned}
$$

At large $L$, the argument of the cosine and the sine in Eqs. (21) and (22) is of order $\sim L$ and those functions become very rapidly oscillating. We have in any event that $\hat{n}^{2}=1$, so that for vanishing $k_{y}$

$$
\left.V_{\text {eff }}(x)\right|_{k_{y}=0}=\left(A_{y}(x)-\sigma_{z} A_{x}(x) \hat{m}\right)^{2}-A_{x}^{2}(x) .
$$

The last term in (24) acts as a confining potential, while the first term exerts the opposite effect. Given that $A_{x}=-\left(V_{A B^{\prime}}+\right.$ $\left.V_{B A^{\prime}}\right) / 2$, we can anticipate a tendency of the effective gauge field to localize low-energy states in the regions where the interlayer potentials $V_{A B^{\prime}}$ and $V_{B A^{\prime}}$ are not negligible. We have moreover to bear in mind that $A_{y}=\left(V_{A B^{\prime}}-V_{B A^{\prime}}\right) / 2$, so that this component becomes small when $V_{A B^{\prime}} \sim V_{B A^{\prime}}$. This means that, at $k_{y}=0$, the low-energy states must be preferentially confined in the interface between $A B$ and $B A$ stacking.

A similar conclusion can be reached in a more straightforward way when $\varepsilon \approx 0$ (at $k_{y}=0$ ). Then we have from the Hamiltonian (8) (neglecting again for simplicity the scalar potential)

$$
\begin{gathered}
-i \partial_{x} \psi_{A}^{(1)}+V_{B A^{\prime}} \psi_{A^{\prime}}^{(2)} \approx 0, \\
V_{A B^{\prime}}^{\star} \psi_{A}^{(1)}-i \partial_{x} \psi_{A^{\prime}}^{(2)} \approx 0
\end{gathered}
$$

and similar equations for $\psi_{B}^{(1)}, \psi_{B^{\prime}}^{(2)}$. At large $L$, we get, for instance,

$$
-\partial_{x}^{2} \psi_{A}^{(1)}-V_{B A^{\prime}} V_{A B^{\prime}}^{\star} \psi_{A}^{(1)} \approx 0,
$$

which shows that low-energy modes have to be confined at the interfaces of the sheared bilayer where $V_{B A^{\prime}} V_{A B^{\prime}}^{\star}$ gets larger values.

We recall that the model of effective non-Abelian gauge fields has been already used to investigate the behavior of the low-energy bands in sheared bilayers with alternating $A A-$ $A B-B A$ stacking [12]. In the case of an infinite superlattice with such a stacking sequence, it has been shown that the model implies indeed the existence of four almost flat bands per Dirac valley. We note that this is in agreement with the existence of eight approximately flat bands in the low-energy picture shown in Fig. 2(a), taking into account that the plot results from the superposition of the bands from the two valleys at $K$ and $K^{\prime}$ points (which have both momentum $k_{y}=0$ ).

Here we pay attention specifically to the confining properties of the gauge field arising from the $A B-B A$ domain wall, which are more properly described in the sheared bilayer with open boundary conditions. In the plot of Fig. 2(b), the number of approximately flat bands near $\varepsilon=0$ is reduced to four, which is a consequence of switching off any confining effect about $A A$ stacking after cutting the bilayer in that region. To illustrate the localization of the states, we have represented in Fig. 3 a sequence of the local density of states from the four approximately flat bands closer to $\varepsilon=0$ in the plot of Fig. 2(b). We observe that the maxima in the local density shift away from the intermediate region connecting $A B$ and $B A$ stacking

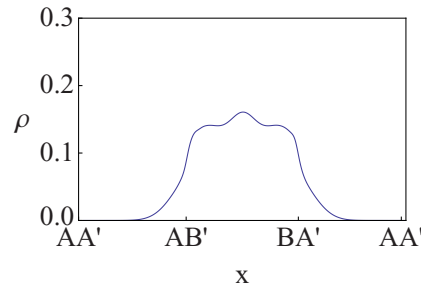

(a)

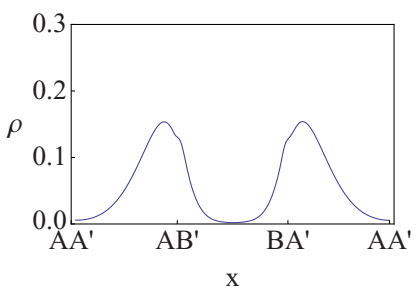

(c)

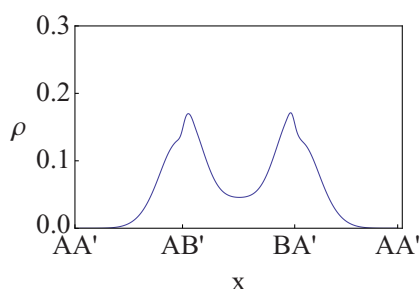

(b)

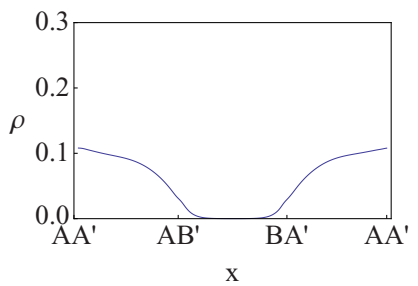

(d)
FIG. 3. Local density from the states in the four low-energy bands closer to $\varepsilon=0$ in Fig. 2(b), at respective momenta [from (a) to (d)] $k_{y}=0.01 \pi / a, 0.014 \pi / a, 0.019 \pi / a$, and $0.023 \pi / a$ ( $a$ being the C-C distance).

as $k_{y}$ grows. This is consistent with the expression of the potential (23), as it can be seen that the term $-A_{y}^{2}$ is effectively switched on when the momentum $k_{y}$ starts deviating from zero. Since $A_{y}=\left(V_{A B^{\prime}}-V_{B A^{\prime}}\right) / 2$, that reinforces the confinement already induced by the term $-A_{x}^{2}$, but moving it towards the regions where either $V_{A B^{\prime}}$ or $V_{B A^{\prime}}$ have the largest strength.

As observed also from Fig. 3, the local density of states becomes suppressed in the region between $A B$ and $B A$ stacking beyond a certain momentum $k_{y}$, when this reaches the dispersive part of the bands with lowest energy in Fig. 2(b). In that regime, the modes in the linear branches of such bands can be seen as edge states that are confined to the region where the gauge field strength fades away. This draws a consistent picture overall, in which the four approximately flat bands in Fig. 2(b) can be interpreted as the zeroth Landau level characteristic of a Dirac fermion field, besides other low-energy bands which resemble those appearing in graphene lattices placed under a real periodic magnetic field [27].

\section{B. Repulsion from non-Abelian gauge fields in strained bilayers}

We can adopt a similar large- $L$ approach to explain the lowenergy properties of the strained bilayers with constant $u_{y y}^{(a)} \neq$ 0 . In this case the interlayer potentials $V_{A A^{\prime}}, V_{A B^{\prime}}$ and $V_{B A^{\prime}}$ become functions only of the $y$ variable, and we can solve the eigenvalue problem (13) by introducing spinor wavefunctions of the form

$$
\Psi(\mathbf{r})=e^{i k_{x} x} \chi(y) .
$$

In the limit of large $L$, we get the eigenvalue equation

$$
\begin{aligned}
& v_{F}^{2}\left(\left(-i \partial_{y}-A_{y}(y) \tau_{2}\right)^{2}+\left(k_{x}-A_{x}(y) \tau_{1}\right)^{2}\right. \\
& \left.-2 \sigma_{z} \tau_{3} A_{x}(y) A_{y}(y)\right) \chi(y)=\varepsilon^{2} \chi(y) .
\end{aligned}
$$

We may obtain again an effective Schrödinger equation by applying a gauge transformation $\chi=U \tilde{\chi}$, 
with

$$
\begin{gathered}
U=\exp \left(i \theta^{\prime}(y) \tau_{2}\right), \\
\theta^{\prime}(y)=\int^{y} d s A_{y}(s) .
\end{gathered}
$$

Equation (29) is converted then into

$$
\begin{aligned}
& v_{F}^{2}\left(-\partial_{y}^{2}+U^{\dagger}\left(k_{x}-A_{x}(y) \tau_{1}\right)^{2} U\right. \\
& \left.\quad-2 \sigma_{z} U^{\dagger} \tau_{3} A_{x}(y) A_{y}(y) U\right) \tilde{\chi}(y)=\varepsilon^{2} \tilde{\chi}(y) .
\end{aligned}
$$

Operating with the Pauli matrices, we arrive at

$$
\begin{aligned}
& v_{F}^{2}\left(-\partial_{y}^{2}+\left(k_{x}-A_{x}(y) \hat{n}^{\prime}\right)^{2}+\left(A_{x}(y)-\sigma_{z} A_{y}(y) \hat{m}^{\prime}\right)^{2}\right. \\
& \left.\quad-A_{x}^{2}(y)-A_{y}^{2}(y)\right) \tilde{\chi}(y)=\varepsilon^{2} \tilde{\chi}(y),
\end{aligned}
$$

where we have the matrices with unit square

$$
\begin{gathered}
\hat{m}^{\prime}=\cos \left(2 \theta^{\prime}(y)\right) \tau_{3}+\sin \left(2 \theta^{\prime}(y)\right) \tau_{1}, \\
\hat{n}^{\prime}=-\sin \left(2 \theta^{\prime}(y)\right) \tau_{3}+\cos \left(2 \theta^{\prime}(y)\right) \tau_{1} .
\end{gathered}
$$

The expression (33) can be then interpreted as a Schrödinger equation, providing an effective potential

$$
\begin{aligned}
V_{\mathrm{eff}}^{\prime}(y)= & \left(k_{x}-A_{x}(y) \hat{n}^{\prime}\right)^{2}+\left(A_{x}(y)-\sigma_{z} A_{y}(y) \hat{m}^{\prime}\right)^{2} \\
& -A_{x}^{2}(y)-A_{y}^{2}(y) .
\end{aligned}
$$

For very large $L$, the cosine and the sine in $\hat{m}^{\prime}$ and $\hat{n}^{\prime}$ oscillate very fast, producing ups and downs that average to zero. We have however that $\left(\hat{n}^{\prime}\right)^{2}=1$, and we get for vanishing $k_{x}$

$$
\left.V_{\mathrm{eff}}^{\prime}(y)\right|_{k_{x}=0}=\left(A_{x}(y)-\sigma_{z} A_{y}(y) \hat{m}^{\prime}\right)^{2}-A_{y}^{2}(y) .
$$

The only source of confinement at $k_{x}=0$ may come from the term $-A_{y}^{2}$ in (37), but that vanishes for $V_{A B^{\prime}}=V_{B A^{\prime}}$. In the regions where either $V_{A B^{\prime}}$ or $V_{B A^{\prime}}$ have large strength, confinement is otherwise compensated by the repulsion exerted by $A_{x}$ in the first term at the right-hand side of (37). It becomes clear then that the effective gauge field accounting for the interlayer coupling around $A B$ and $B A$ stacking cannot lead generically to confinement of low-energy states in the case of the strained bilayer.

Concentrating on the low-energy regime with $\varepsilon \approx 0$ at $k_{x}=$ 0 , it can be shown more directly that the interlayer potentials induce an effect of repulsion at the interface between $A B$ and $B A$ stacking in the strained bilayer. From the Hamiltonian (8), we get in these conditions

$$
\begin{aligned}
\partial_{y} \psi_{A}^{(1)}+V_{B A^{\prime}} \psi_{A^{\prime}}^{(2)} & \approx 0, \\
V_{A B^{\prime}}^{\star} \psi_{A}^{(1)}+\partial_{y} \psi_{A^{\prime}}^{(2)} & \approx 0
\end{aligned}
$$

and similar equations for $\psi_{B}^{(1)}, \psi_{B^{\prime}}^{(2)}$. In the limit of large $L$, possible low-energy states should correspond therefore to solutions of the equation

$$
-\partial_{y}^{2} \psi+V_{B A^{\prime}} V_{A B^{\prime}}^{\star} \psi \approx 0
$$

As long as $V_{A B^{\prime}}$ and $V_{B A^{\prime}}$ provide a smooth representation of the interlayer tunneling, we must have $V_{B A^{\prime}} V_{A B^{\prime}}^{\star}>0$ in the region between $A B$ and $B A$ stacking. This means

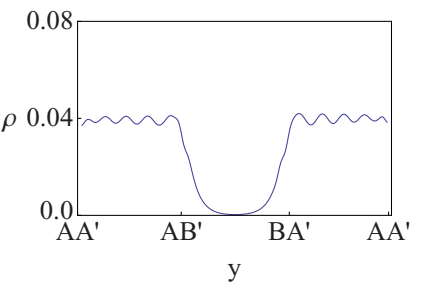

(a)

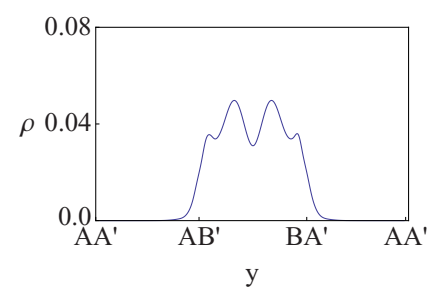

(c)

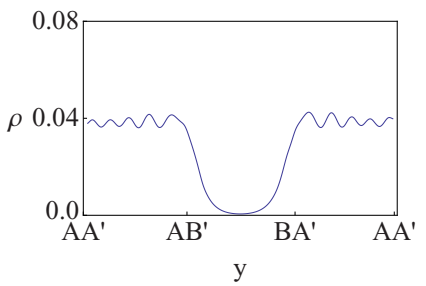

(b)

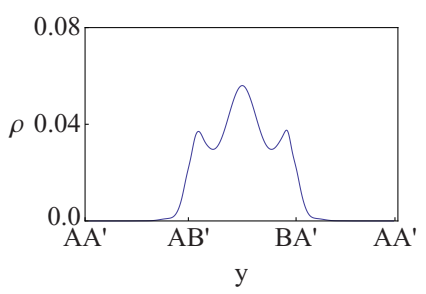

(d)
FIG. 4. (a), (b) Local density from the states with $k_{x}=0$ in the linear branch close to zero energy (a) and at the bottom of the quadratic band right above zero energy (b) in the band structure shown in Fig. 2(d). (c), (d) Local density from the states with $k_{x}=0.014 \pi / a$ ( $a$ being the C-C distance) at the top of the quadratic band right below zero energy (c) and at the top of the quadratic band right above zero energy (d) appearing to the right in the band structure of Fig. 2(d).

that no low-energy states can be bound by the potential in (40), implying that no low-energy states can arise from confinement due to the non-Abelian gauge field in the strained bilayers.

The description in terms of the effective gauge field can also account for the transition to a different regime when we consider momenta $k_{x} \neq 0$. To illustrate this effect, we have represented in Fig. 4 the local density of states from different bands at vanishing as well as nonvanishing momentum $k_{x}$. As shown in the figure, the local density of states with $k_{x}=0$ is in general suppressed in the region between $A B$ and $B A$ stacking, in accordance with the above arguments. The plots of the local density of states with $k_{x} \neq 0$ [taken from the parabolic bands shown in Fig. 2(d)] display instead a clear confinement in the region mediating $A B$ and $B A$ stacking. This is consistent again with the above description, since shifting the momentum $k_{x}$ away from zero amounts to switching on the term $-A_{x}^{2}$ in the effective potential (36), leading to attraction to the regions where $V_{A B^{\prime}}$ and $V_{B A^{\prime}}$ have larger strength.

We observe that the effects of the non-Abelian gauge field in the strained bilayer are radically different to those in the sheared bilayer. Looking at Figs. 3 and 4, it may seem that the patterns are inverted when passing from one type of bilayer to the other. However, it has to be stressed that the confinement seen in Figs. 4(c) and 4(d) in the intermediate region between $A B$ and $B A$ stacking corresponds to states in parabolic bands whose energy is in general not small. This phenomenon is very different to the localization that is displayed by the states in the approximately flat bands developed at low energies by the sheared bilayers. It is in that case that the effects of the non-Abelian gauge field can be assimilated to those of a real Abelian gauge field, regarding in particular the formation of flat bands from localized states. 


\section{Snake states in sheared graphene bilayers}

We want to make contact at this point with the results of previous studies dealing with stacking domain walls in graphene bilayers. These analyses have mainly focused on the description of abrupt boundaries between different stacking regions, or when there is also a difference in gate voltage between the graphene layers [20,21]. In those cases, the main finding has been that several linear branches appear within the gap in the electronic spectrum, connecting the valence and conduction bands of the graphene bilayer. It has been shown that these low-energy branches arise as a topological effect, which has its origin in the mismatch in the Berry curvature and Chern number of the regions connected by the stacking domain wall $[20,21,23]$. In the case of smooth modulations of the stacking pattern (with no transverse electric field), we are going to see that there are similar low-energy branches, which can be understood here as a result of the inversion in the orientation of the gauge field strength at the interface between different stacking regions.

We have already mentioned that, in the limit of large $L$, the field strength of the non-Abelian gauge field is dominated by the last term in Eq. (14). In the case of the relevant $x y$ component, we get

$$
\hat{F}_{x y} \approx 2 A_{x}(x) A_{y}(x) \tau_{3},
$$

which vanishes at the boundary where $V_{A B^{\prime}}=V_{B A^{\prime}}$. In cases where one has a modulated magnetic field, the lines corresponding to vanishing field strength give rise to effective boundaries in the electron system where new edge states may appear. These are the so-called snake states, which have been found in a number of situations where the spatial modulation leads to an inversion in the orientation of the magnetic field [27-29]. In our graphene bilayers, we have also signatures of snakes states, which are already present in the low-energy regime of band structures like those shown in Figs. 2(a) and 2(b). One has however to make a zoom around the approximately flat bands, in order to have an enhanced view of the relevant features. These are clearly resolved in Fig. 5(a). We observe there the presence of low-energy branches with linear crossing, which are the precursor of the linear branches between valence and conduction bands already found in the case of gated graphene bilayers. The plot of the local density from the states at the crossing point between the linear branches, represented in Fig. 5(b), shows in a clear way

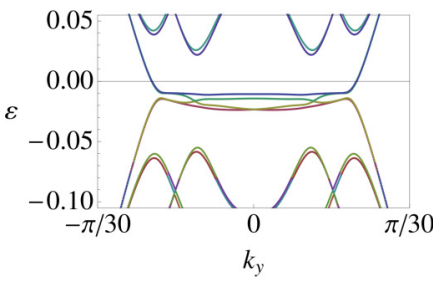

(a)

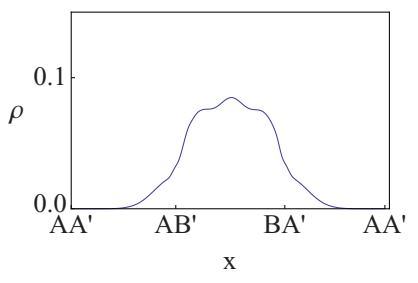

(b)
FIG. 5. (a) Zoom view of the low-energy part of the band structure shown in Fig. 2(b). The energy $\varepsilon$ is given in $\mathrm{eV}$ and the momentum in units of the inverse of the C-C distance. (b) Plot of the local density from the states at the crossing of the linear branches within the approximately flat bands in (a). the confinement of the states in the region between $A B$ and $B A$ stacking.

The crossing of the low-energy bands seen in Fig. 5(a) is indeed a direct consequence of the modulated gauge field arising from the $A B-B A$ stacking pattern. This connection can be established more precisely by analyzing the effective gauge field model, adopting a simplified formulation in which the scalar potential $V_{A A^{\prime}}$ is switched off. The spectrum of the Hamiltonian (11) has particle-hole symmetry, which means that the crossing of low-energy linear branches can be characterized from the presence of zero-energy modes. Going back to the Hamiltonian (8), such modes must arise as solutions of the system (assuming that the interlayer potentials are real)

$$
-i \partial_{x}\left(\begin{array}{l}
\chi^{(1)} \\
\chi^{(2)}
\end{array}\right)=\left(\begin{array}{cc}
i k_{y} & -V_{A B^{\prime}} \\
-V_{B A^{\prime}} & i k_{y}
\end{array}\right)\left(\begin{array}{l}
\chi^{(1)} \\
\chi^{(2)}
\end{array}\right) .
$$

Equation (42) can be formally integrated, leading in matrix form to

$$
\begin{aligned}
\left(\begin{array}{l}
\chi^{(1)}(x) \\
\chi^{(2)}(x)
\end{array}\right)= & \operatorname{Pexp}\left\{i \int_{0}^{x} d s\left[i k_{y} \mathbb{1}+A_{x}(s) \tau_{1}-i A_{y}(s) \tau_{2}\right]\right\} \\
& \times\left(\begin{array}{l}
\chi^{(1)}(0) \\
\chi^{(2)}(0)
\end{array}\right),
\end{aligned}
$$

where "Pexp" means that the matrix is built from the product of exponentials of the differential line elements. While the formal expression (43) shows that zero-energy modes may exist, we note that they are bound to satisfy a quantization condition, which arises from the boundary conditions on the wave functions. In the case of bilayers where those are imposed for instance at $x=0$ and $x=L$, that amounts to enforce the constraint

$$
\operatorname{Pexp}\left\{i \int_{0}^{L} d s\left[i k_{y} \mathbb{1}+A_{x}(s) \tau_{1}-i A_{y}(s) \tau_{2}\right]\right\}=\mathbb{1}
$$

or, less restrictively, the unitarity of the exponential operator in (44).

These considerations can be illustrated most easily in the sheared bilayers with periodic boundary conditions. In that case, sensible results can be already obtained by taking a single-harmonic approximation for the interlayer potentials, which are then represented as $V_{A B^{\prime}}(x)=$ $\left(\lambda / v_{F}\right)[1+2 \cos (2 \pi x / L-\pi / 3)]$ and $V_{B A^{\prime}}(x)=\left(\lambda / v_{F}\right)[1+$ $2 \cos (2 \pi x / L+\pi / 3)][12]$. By introducing these expressions in (44), it can be seen that the boundary condition constrains indeed the appearance of the zero modes. For $k_{y}=0$, one finds for instance that the quantization condition (44) is satisfied for values of $L$ such that $\lambda L / v_{F}=2 \pi n$, with integer $n$. When $L$ does not correspond to any of these values, it is still possible to find zero modes for $k_{y} \neq 0$. This is the instance which is represented in Fig. 6, displaying the low-energy bands in the effective gauge field model for $\lambda=0.1 \mathrm{eV}$ and $\lambda L / v_{F}=6.2 \pi$. The results from this simple approximation show to be consistent with the low-energy features found in the tight-binding calculation, clarifying the origin of the linear crossings in the case of the sheared bilayers.

We remark that snake states appear in the sheared bilayers from the modulation of the effective gauge field, but also as a consequence of the confining character of the interaction that derives from it. This explains that similar states do not 


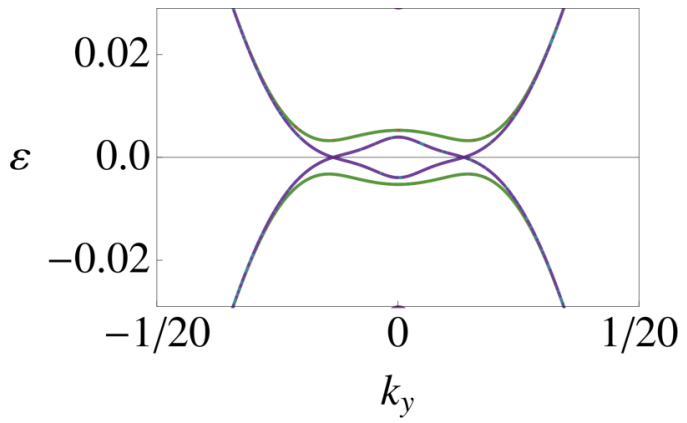

FIG. 6. Zoom view of the low-energy bands for a sheared bilayer in the effective gauge field model, obtained with a single-harmonic approximation to the interlayer potentials for $\lambda=0.1 \mathrm{eV}$ and $\lambda L / v_{F}=6.2 \pi$. The energy $\varepsilon$ is given in $\mathrm{eV}$ and the momentum in units of the inverse of the $\mathrm{C}-\mathrm{C}$ distance.

arise in the case of the strained bilayers. As can be seen in the band structures shown in Figs. 2(c) and 2(d), low-energy linear branches also exist in those systems. However, their character is very different to those corresponding to snake states in the sheared bilayers. This can be already appreciated from the shape of a typical state in the linear branches of the strained bilayers shown in Fig. 4(a), which is a reflection of the repulsive interaction expelling the electronic density from the region between $A B$ and $B A$ stacking.

\section{CONCLUSION}

We have seen that the action of strain and shear may lead to graphene bilayers which are quite different from the point of view of their low-energy electronic properties. The mismatch in the registry of the bilayers produces characteristic Moiré patterns which can look very similar when observed from a wide perspective, but may in fact correspond to quite different band structures. We have ascribed the different electronic behavior to the effect of a fictitious non-Abelian gauge field, which can mimic the modulation from $A B$ to $B A$ stacking induced by strain or shear in the bilayers.

We have carried out a comparative analysis of two representative bilayers with quite different band structures, extending the analyses of previous studies about abrupt stacking domain walls in a transverse electric field. We have thus seen that strained and sheared bilayers show a complementary behavior, as the low-energy states of the former are in general expelled from the region between $A B$ and $B A$ stacking, while those of the sheared bilayers tend to be confined around that interface. In our effective gauge field model, this can be understood from the fact that the non-Abelian gauge field may act as a repulsive interaction (in the case of the strained bilayers) or as a confining interaction leading to localization (in the case of the sheared bilayers).

The present study becomes pertinent as there have been already several experimental observations at the atomic scale of stacking domain walls in graphene bilayers. These adopt in general the form of smooth modulations in the stacking sequence, which can be conveniently described in the framework of our continuum approximation. The sample reported in Ref. [18] seems to correspond for instance to a sheared bilayer with a sequence of $A B-B A$ stacking, showing clear signatures of low-energy electronic states around the stacking domain wall. This is in agreement with the phenomenology that we expect from a sheared bilayer. It is quite likely that more experimental samples of strained or sheared bilayers can be observed in the future, which may allow one to confirm the correspondence we have drawn between the atomic arrangement and the low-energy electronic properties of the bilayers.

From a theoretical point of view, we have elucidated the possibility that a non-Abelian gauge field can give rise to a large degeneracy of low-energy states, acting much in the same way as a conventional gauge field in the quantum Hall regime. The approximately flat low-energy bands we have found in the sheared bilayers are indeed the analog of the zeroth Landau level that arises in Dirac systems under a strong magnetic field. The lateral linear branches that are seen at each side in Figs. 2(a) and 2(b) correspond to edge states, that are here localized at the region where the gauge field strength fades away. The magnitude of the effective field strength can be easily estimated from the extension of the approximately flat bands in Figs. 2(a) and 2(b), giving values of the order of several tens of tesla. The experimental signatures of such strong effective fields should be then quite robust, leading to a phenomenology susceptible of being observed in suitably distorted bilayer samples.

\section{ACKNOWLEDGMENT}

We acknowledge financial support from MINECO (Spain) through Grant No. FIS2014-57432-P.
[1] K. Novoselov, A. Geim, S. Morozov, D. Jiang, M. Katsnelson, I. Grigorieva, S. Dubonos, and A. Firsov, Nature (London) 438, 197 (2005).

[2] Y. Zhang, Y. Tan, H. Stormer, and P. Kim, Nature (London) 438, 201 (2005).

[3] A. H. Castro Neto, F. Guinea, N. M. R. Peres, K. S. Novoselov, and A. K. Geim, Rev. Mod. Phys. 81, 109 (2009).

[4] M. I. Katsnelson, K. S. Novoselov, and A. K. Geim, Nat. Phys. 2, 620 (2006).
[5] J. González, F. Guinea, and M. Vozmediano, Nucl. Phys. B 406, 771 (1993).

[6] F. Guinea, M. Katsnelson, and A. Geim, Nat. Phys. 6, 30 (2009).

[7] S. V. Morozov, K. S. Novoselov, M. I. Katsnelson, F. Schedin, L. A. Ponomarenko, D. Jiang, and A. K. Geim, Phys. Rev. Lett. 97, 016801 (2006).

[8] J. L. Mañes, Phys. Rev. B 76, 045430 (2007).

[9] N. Levy, S. A. Burke, K. L. Meaker, M. Panlasigui, A. Zettl, F. Guinea, A. H. Castro Neto, and M. F. Crommie, Science 329, 544 (2010). 
[10] M. Mucha-Kruczyński, I. L. Aleiner, and V. I. Fal'ko, Phys. Rev. B 84, 041404(R) (2011).

[11] Y.-W. Son, S.-M. Choi, Y. P. Hong, S. Woo, and S.-H. Jhi, Phys. Rev. B 84, 155410 (2011).

[12] P. San-José, J. González, and F. Guinea, Phys. Rev. Lett. 108, 216802 (2012).

[13] E. Mariani, A. J. Pearce, and F. von Oppen, Phys. Rev. B 86, 165448 (2012).

[14] For the appearance of effective magnetic fields in onedimensional graphene superlattices, see also J. Sun, H. A. Fertig, and L. Brey, Phys. Rev. Lett. 105, 156801 (2010).

[15] J. S. Alden, A. W. Tsen, P. Y. Huang, R. Hovden, L. Brown, J. Park, D. A. Muller, and P. L. McEuen, Proc. Natl. Acad. Sci. USA 110, 11256 (2013).

[16] B. Butz, C. Dolle, F. Niekiel, K. Weber, D. Waldmann, H. B. Weber, B. Meyer, and E. Spiecker, Nature (London) 505, 533 (2014).

[17] L. Ju, Z. Shi, N. Nair, Y. Lv, C. Jin, J. Velasco, Jr., C. OjedaAristizabal, H. A. Bechtel, M. C. Martin, A. Zettl, J. Analytis, and F. Wang, Nature (London) 520, 650 (2015).

[18] L.-J. Yin, H. Jiang, J.-B. Qiao, and L. He, Nat. Commun. 7, 11760 (2016)
[19] S. Hattendorf, A. Georgi, M. Liebmann, and M. Morgenstern, Surf. Sci. 610, 53 (2013).

[20] F. Zhang, A. H. MacDonald, and E. J. Mele, Proc. Natl. Acad. Sci. USA 110, 10546 (2013).

[21] A. Vaezi, Y. Liang, D. H. Ngai, L. Yang, and E.-A. Kim, Phys. Rev. X 3, 021018 (2013).

[22] I. Martin, Ya. M. Blanter, and A. F. Morpurgo, Phys. Rev. Lett. 100, 036804 (2008).

[23] J. Jung, F. Zhang, Z. Qiao, and A. H. MacDonald, Phys. Rev. B 84, 075418 (2011).

[24] See also M. Van der Donck, C. De Beule, B. Partoens, F. M. Peeters, and B. Van Duppen, 2D Mater. 3, 035015 (2016).

[25] A. A. Maarouf, C. L. Kane, and E. J. Mele, Phys. Rev. B 61, 11156 (2000).

[26] C. Itzykson and J. B. Zuber, Quantum Field Theory (McGrawHill, New York, 1985), Chap. 12.

[27] E. Perfetto, J. González, F. Guinea, S. Bellucci, and P. Onorato, Phys. Rev. B 76, 125430 (2007).

[28] H. Ajiki and T. Ando, J. Phys. Soc. Jpn. 62, 1255 (1993); 65, 505 (1996).

[29] H.-W. Lee and D. S. Novikov, Phys. Rev. B 68, 155402 (2003). 